\title{
Practical Application of Morphology Nomenclature for Pharmaceutical Materials
}

\author{
L. M. DiMemmo, ${ }^{*}$ L. Capecci, ${ }^{* *}$ J. Thomas, ${ }^{* *}$ B. Sarsfield* \\ * Bristol-Myers Squibb Company, 1 Squibb Drive, New Brunswick, NJ 08903-0191 \\ ** Drexel University, 245 N. $15^{\text {th }}$ Street, Philadelphia, PA 19102
}

It is well known that particle morphology can influence pharmaceutical formulation processes such as mixing, powder packing, milling, filtration, tabletting, dissolution and more. Therefore, particle shape is a critical variable and understanding it is essential for the development of an active pharmaceutical ingredient and drug product. Microscopy is the technique of choice by scientists from a variety of disciplines to characterize particle shape. A problem arises when each scientific discipline uses different terms to describe similar materials. This happens for two main reasons. First, there are a vast number of terms in the literature to choose from. Second, although the use of axial dimensions to describe particle shape has been reported in the literature, particle dimension ranges are not necessarily well defined [1].

If particle shape is not described in a consistent manner, it is difficult to determine if particle shape is different when comparing samples. To address this issue, we developed a simplified set of axial dimensions to describe particle shape and an optical microscopy test to monitor particle shape using the axial dimension scheme. The axial dimension scheme uses a manual measurement that complies directly with USP guidelines for measuring morphology [2]. The axial dimension system is a two step approach using axial dimension ratios and well defined ranges to provide consistency in the descriptors used for several particle shapes. With the axial dimension scheme, number and shape are related by starting with a shape, i.e. needle, plate. This is followed by assigning a number to the shape.

In this work, image analysis measurements were evaluated to see how they fit with the axial dimension scheme. Using image analysis (IA) makes it possible to automate measurements so that a large number of particles can be analyzed quickly. With image analysis, number and shape are related by starting with a number, i.e. feature measurement and assigning a shape such as needle or plate to the number. Samples were selected to represent different particle shapes such as needles, plates and spheres. An image analysis routine was written for each particle shape. The IA routine was run and the particles were mapped. After measuring the axial dimension ratio (ADR), the ADR can be compared to the measurement made by the IA software. The data presented will show how the axial dimension scheme tracks against feature measurements by IA for needles, plates and spheres. It will also demonstrate that selecting the best feature measurement to describe particle shape is shape dependent.

\section{References}

[1] Aldrich, D. Scott, Smith, Mark A.: Applied Spectroscopy Reviews, 34(4), 275-327, (1999). [2] USP 27, NF 22, United States Pharmacopeial Convention, Inc., Rockville, MD, <776>, (2004). 


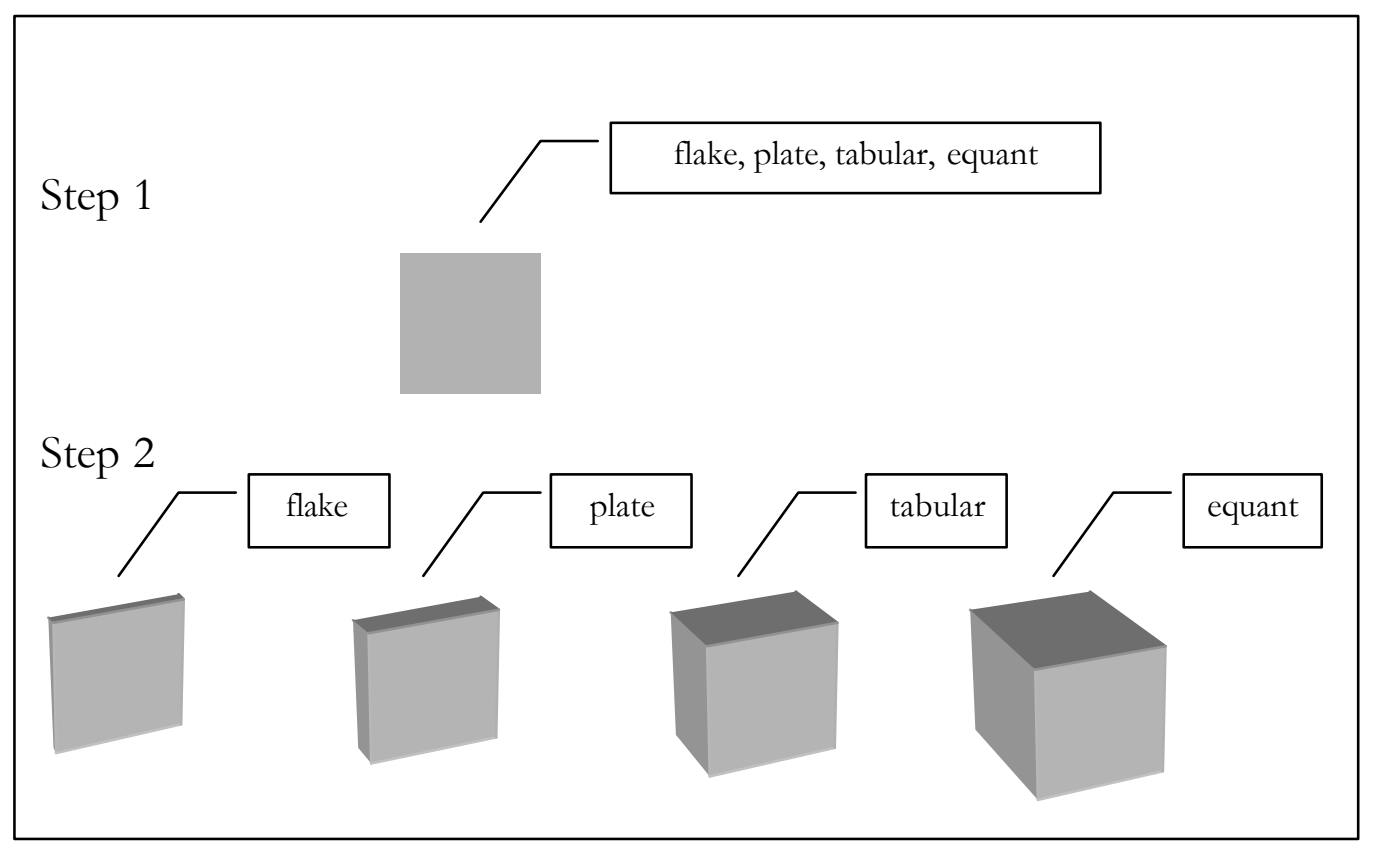

Figure 1. Drawing representing the two step axial dimension system for classifying particle shape. Step 1 shows a particle with a width:length ratio of 1:1. In Step 2, width:length is combined with thickness:width to further classify particle shape.
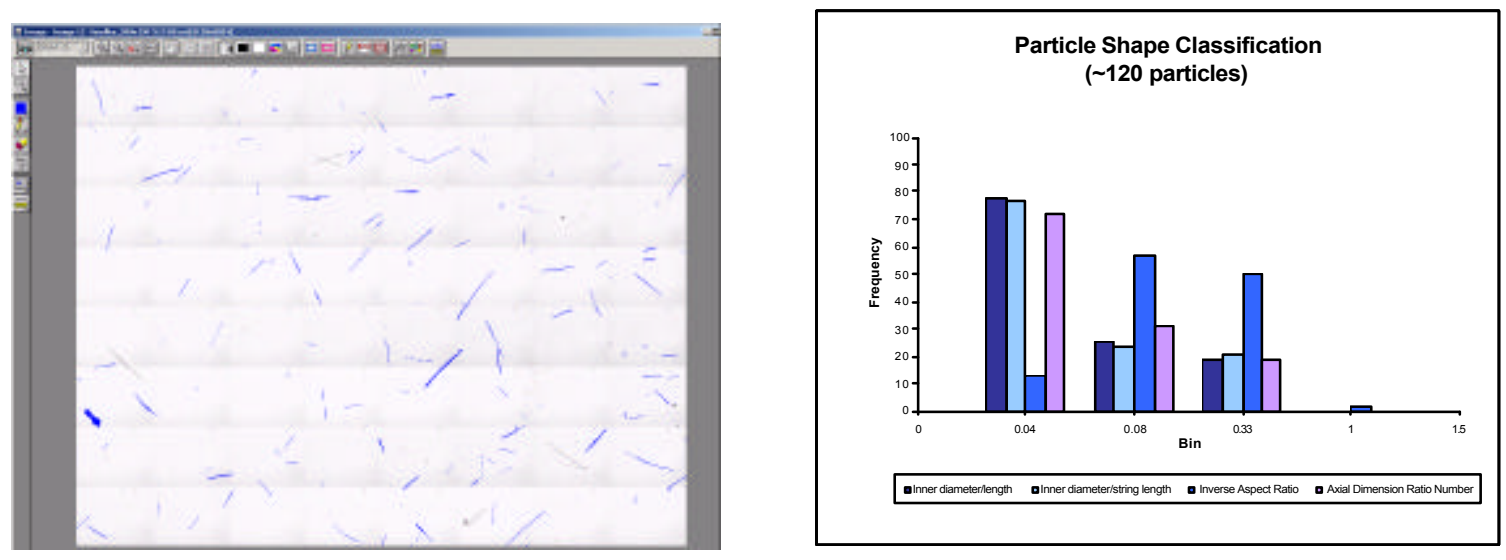

Figure 2. Particle map for needles (left) and graph (right) showing particle shape classification by IA (blue bars) and ADR (lavender bar) for 120 particles of this needle sample. 